\title{
ISECG Global Exploration Roadmap: A Stepwise Approach to Deep Space Exploration
}

\author{
Roland Martinez ${ }^{1}$ \\ NASA Johnson Space Center, Houston, TX, 77058 \\ and \\ Kandyce Goodliff ${ }^{2}$ \\ NASA Langley Research Center, Hampton, VA, 23681 \\ and \\ Ryan Whitley ${ }^{3}$ \\ NASA Johnson Space Center, Houston, TX, 77058
}

\begin{abstract}
In 2011, ISECG released the Global Exploration Roadmap (GER), advancing the "Global Exploration Strategy: The Framework for Coordination" by articulating the perspectives of participating agencies on exploration goals and objectives, mission scenarios, and coordination of exploration preparatory activities. The GER featured a stepwise development and demonstration of capabilities ultimately required for human exploration of Mars.

In 2013 the GER was updated to reflect the ongoing evolution of agency's exploration policies and plans, informed by individual agency and coordinated analysis activities that are relevant to various elements of the GER framework as well as coordinated stakeholder engagement activities. For this release of version 2 of the GER in the mid 2013 timeframe, a modified mission scenario is presented, more firmly reflecting the importance of a stepwise evolution of critical capabilities provided by multiple partners necessary for executing increasingly complex missions to multiple destinations and leading to human exploration of Mars. This paper will describe the updated mission scenario, the changes since the release of version 1, the mission themes incorporated into the scenario, and risk reduction for Mars missions provided by exploration at various destinations.
\end{abstract}

\section{Introduction}

$\mathrm{T}$ HE International Space Exploration Coordination Group (ISECG) was established in response to "The Global Exploration Strategy (GES): The Framework for Coordination" developed by fourteen space agencies ${ }^{4}$ and released in May 2007. This GES Framework Document recognizes that preparing for human space exploration is a stepwise process, starting with basic knowledge and culminating in a sustained human presence in space. In 2011, ISECG released the Global Exploration Roadmap (GER) ${ }^{\mathrm{i}}$, advancing the GES by articulating the perspectives of participating agencies on exploration goals and objectives, mission scenarios, and coordination of exploration preparatory activities. Two feasible pathways for human exploration were presented after International Space

\footnotetext{
${ }^{1}$ Chair, ISECG International Architecture Working Group, Exploration Mission Systems Office, JSC/YX.

${ }^{2}$ Aerospace Engineer, Space Mission Analysis Branch, LaRC/MS 462, AIAA Member.

${ }^{3}$ Aerospace Engineer, Exploration Mission Systems Office, JSC/YX, AIAA Member

${ }^{4}$ In alphabetical order: ASI (Italy), BNSC - now UKSA (United Kingdom), CNES (France), CNSA (China), CSA (Canada), CSIRO (Australia), DLR (Germany), ESA (European Space Agency), ISRO (India), JAXA (Japan), KARI (Republic of Korea), NASA (United States of America), NSAU (Ukraine), Roscosmos (Russia). "Space Agencies" refers to government organizations responsible for space activities.
}

American Institute of Aeronautics and Astronautics 
Station (ISS) - "Asteroid Next" and "Moon Next". They differ primarily with regard to the sequence of sending humans to the Moon and asteroids, and each reflects a stepwise development and demonstration of capabilities ultimately required for human exploration of Mars.

Periodic updates of the GER will reflect the ongoing evolution of space agencies' exploration policies and plans, informed by agency individual and coordinated analysis activities that are relevant to various elements of the GER framework as well as coordinated stakeholder engagement activities. For the release of version 2 of the GER in the mid 2013 timeframe, one mission scenario is presented. It reflects the importance of a stepwise evolution of critical capabilities provided by multiple partners. These capabilities are necessary for executing increasingly complex missions to multiple destinations leading to human exploration of Mars.

This paper will provide a description of the updates of the 2nd version of the GER and highlight the ISECG mission scenario in Section II. Section III will describe the mission scenario changes between version 1 and version 2. The mission themes for the GER will be discussed in Section IV. Section V will describe risk reduction of the mission scenario for human missions to Mars. A summary is presented in Section VI. For more information on the ISECG, please consult the ISECG website at www.globalspaceexploration.org or contact the ISECG Secretariat at: isecg@esa.int.

\section{Mission Scenario}

The ISECG conceptual mission scenario reflects the near-term initiatives in implementing a common strategy, namely: 1) fully utilizing the ISS, 2) continuing efforts to expand on synergies between human and robotic missions, and 3) discovery-driven missions in the lunar vicinity that evolve capabilities and techniques needed for Mars, while enabling discoveries on the Moon and near-Earth asteroids (NEAs). This scenario reflects a coordinated international effort to advance common goals and objectives while enabling interested agencies to pursue their priorities and prepare for critical contributions to human Mars missions. The scenario reflects NASA's priority for pursuing asteroid missions as a step towards advancing critical capabilities needed for future missions to the Mars system. As an international mission scenario, it recognizes that other space agencies favor the Moon as their next human space exploration destination, and can use these missions to demonstrate critical capabilities needed for future Mars surface missions.

Several driving principles ${ }^{\mathrm{i}}$ reflect the characteristics of a sustainable human exploration effort. The principles are highly interdependent, and it is the combination of these principles, rather than any individual one, that drives the development of sustainable mission scenarios. The principles driving the mission scenario are:

- Affordability - Take into account budget constraints

- Exploration Value - Generate public benefits and meet exploration objectives

- International Partnerships - Provide early and sustained opportunities for diverse partners

- Capability Evolution - Execute missions of increasing complexity based on the stepwise development of capabilities

- Human/Robotic Partnership - Maximize synergy between human and robotic missions

- Robustness - Provide for resilience to programmatic and technical challenges.

The mission scenario (Figure 1) reflects missions in the next 25 years which significantly advance exploration objectives on the path to Mars. The scenario reflects a stepwise development and demonstration of capabilities necessary for executing increasingly complex missions, while focusing on discoveries at multiple destinations. Activities in the lunar vicinity and on the lunar surface can enable a variety of objectives related to asteroid and lunar exploration and significantly advance readiness for human exploration of Mars. Ongoing and planned robotic and human missions are critical for preparing for human missions to Mars. The mission scenario includes both human and robotic missions along with multi-destination transportation capabilities, both planned and conceptual, that will support human missions. Potential commercial opportunities are indicated in the mission scenario. The increasing role of private initiatives in low-Earth orbit opens the possibility that similar initiatives may be extensible to missions beyond.

Human missions in the scenario start with current and near-term ISS activities. The ISS provides the opportunity to conduct research and technology demonstrations along with advancing critical exploration capabilities. The next step for humans would be to explore beyond low earth orbit into the lunar vicinity. Missions in the lunar vicinity, such as those to an easily accessible asteroid or an evolvable Deep Space Habitat, could enable discoveries and allow demonstration of the systems on which long-duration missions into deep space must rely. Human missions to the lunar surface could allow demonstrations of planetary exploration capabilities and techniques, while pursuing the highest priority lunar science objectives. Capabilities developed and lessons learned from human exploration of the lunar vicinity could advance the readiness for human Mars missions after 2030. 


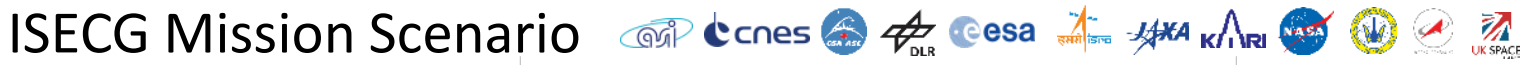

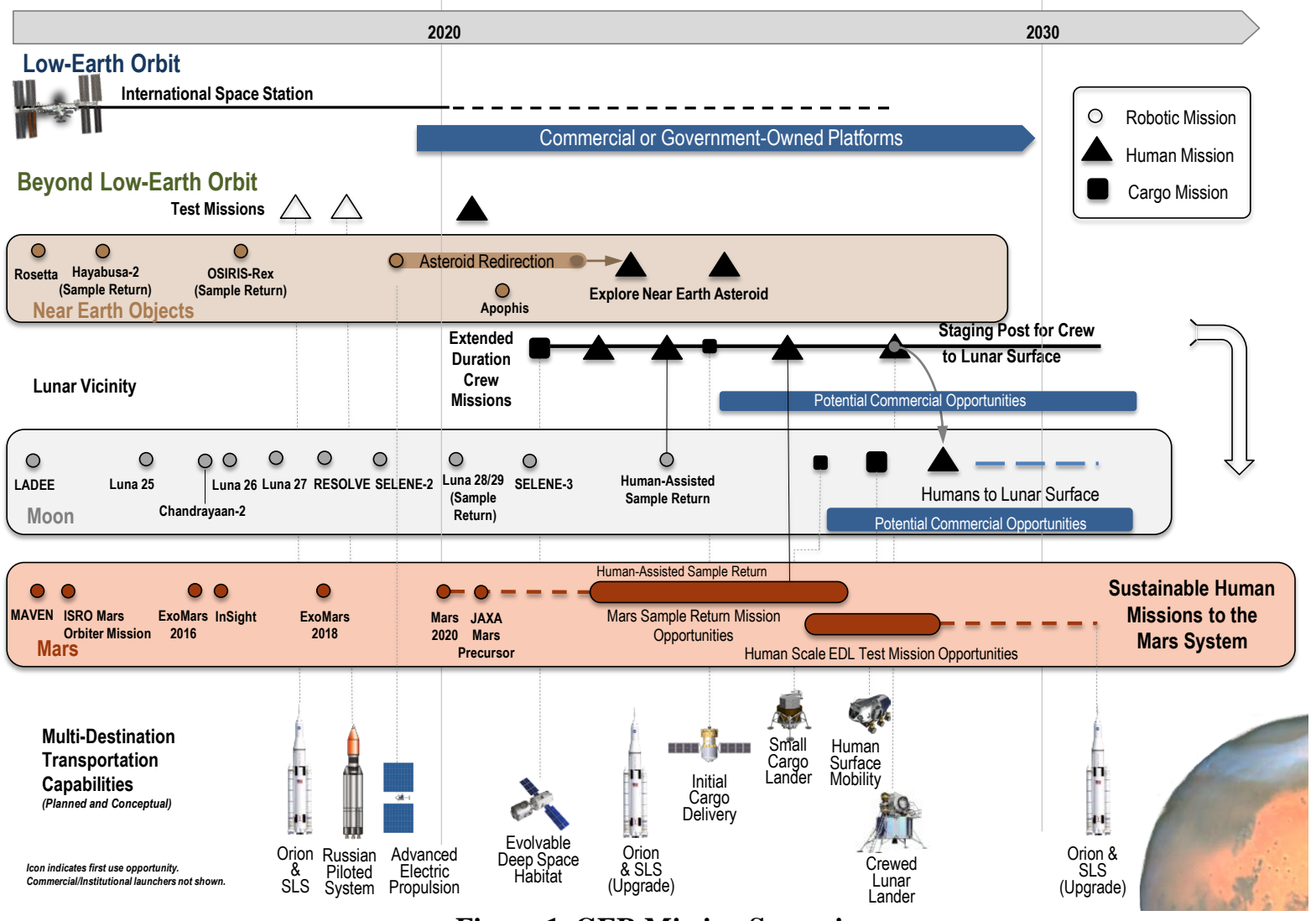

Figure 1. GER Mission Scenario

Crew presence brings human decision-making, adaptability, and resourcefulness, enabling a larger set of science and exploration objectives to be met. Human-assisted sample return and tele-presence represent new and integrated approaches to space exploration with the potential to increase benefits. Mars sample return, a priority for the planetary science community, will bring insights that aid the preparation for eventual human surface missions. Human and robotic mission planners have begun a discussion on whether human presence could enhance sample return, while advancing other human space exploration objectives.

International robotic missions to near-Earth objects, Moon and Mars are reflected in the mission scenario. Future science missions are invaluable resources for providing data that characterize the environments, identify hazards, and assess resources. The knowledge developed from this data will inform the selection of future landing sites, inform the design of new systems, and reduce the risk associated with human exploration. While some data can be obtained through ground-based activities, other data can only be gained in space by remote sensing, in-situ measurements or sample return.

The robotic missions are shown for the three primary destinations - Near-Earth Objects, Moon and Mars. The Near-Earth Object missions focus on sample return, asteroid tracking, and redirecting an asteroid. The lunar missions include sample returns, in-situ resource utilization (ISRU) demonstrations, rovers and landers. The Mars robotic missions include orbiters, landers, and a potential for a sample return mission. Human missions to the surface of Mars will require advances in entry, descent and landing (EDL) capabilities. A human-scale Mars landing test is envisioned, providing an opportunity to also meet other science and precursor mission objectives.

There is a minimum set of capabilities required to support human exploration to multiple destinations beyond low-Earth orbit. These capabilities envisioned are listed in Table 1. Exploration transportation capabilities under development today will enable missions in the lunar vicinity and are designed to be evolvable to support additional missions, such as lunar surface and deep space missions. Other capabilities, such as habitation, surface power, servicing and mobility will be needed over the long term and can be advanced in a stepwise manner to support exploration mission needs. 
Table 1. Major Capabilities

\begin{tabular}{|c|c|c|}
\hline Icon & Capability & Description \\
\hline & NASA Orion & $\begin{array}{l}\text { Crew vehicle capable of delivering a crew to exploration destinations and back to } \\
\text { Earth. }\end{array}$ \\
\hline & $\begin{array}{l}\text { NASA Space Launch } \\
\text { System (SLS) }\end{array}$ & $\begin{array}{l}\text { Launch vehicle with the capability to deliver cargo or crew beyond low-Earth orbit. } \\
\text { Initial capability evolves with advanced boosters and an upper stage to enable } \\
\text { increasingly complex missions with further evolution to support crewed Mars } \\
\text { missions. }\end{array}$ \\
\hline & $\begin{array}{l}\text { Cryogenic Propulsion } \\
\text { Stage (CPS) }\end{array}$ & $\begin{array}{l}\text { Included in SLS evolution plans, an in-space propulsion capability utilizing cryogenic } \\
\text { hydrogen and oxygen as propellants. Could provide additional performance for } \\
\text { missions to the lunar vicinity, lunar surface and Mars. Mission durations will require } \\
\text { long-duration storage of cryogenic propellants. }\end{array}$ \\
\hline & $\begin{array}{l}\text { ROSCOSMOS Next } \\
\text { Generation Space } \\
\text { Launch Vehicle }\end{array}$ & $\begin{array}{l}\text { Launch vehicle with the capability to deliver cargo or crew beyond low-Earth orbit. } \\
\text { The next generation space launch vehicle and the next generation spacecraft together } \\
\text { constitute the Russian Piloted System. }\end{array}$ \\
\hline & $\begin{array}{l}\text { ROSCOSMOS Next } \\
\text { Generation Spacecraft }\end{array}$ & $\begin{array}{l}\text { Crew vehicle capable of delivering a crew to exploration destinations and back to } \\
\text { Earth. }\end{array}$ \\
\hline & $\begin{array}{l}\text { Evolvable Deep Space } \\
\text { Habitat }\end{array}$ & $\begin{array}{l}\text { A human-tended habitat which is evolvable to advance in-space habitation, spacewalk, } \\
\text { and staging capabilities ultimately required for human Mars missions. }\end{array}$ \\
\hline & $\begin{array}{l}\text { Servicing Support } \\
\text { Systems }\end{array}$ & $\begin{array}{l}\text { Systems and tools to enable crew and robots to service space systems and assemble } \\
\text { larger capabilities. These systems can augment the functionality of the Orion and/or } \\
\text { the evolvable Deep Space Habitat. }\end{array}$ \\
\hline & $\begin{array}{l}\text { Cargo Logistics } \\
\text { Delivery Systems }\end{array}$ & $\begin{array}{l}\text { Systems designed to serve as logistic vehicles for resupplying orbital infrastructures in } \\
\text { lunar vicinity with pressurized and unpressurized payloads. }\end{array}$ \\
\hline & Small Cargo Lander & $\begin{array}{l}\text { System designed to deliver robotics and cargo on the lunar surface to meet lunar } \\
\text { exploration objectives. }\end{array}$ \\
\hline & Crewed Lunar Lander & $\begin{array}{l}\text { Human-rated lunar lander that may have attributes of reusability, may be composed of } \\
\text { two or more stages, and delivers crew and cargo to the lunar surface. }\end{array}$ \\
\hline & Lunar Surface Elements & $\begin{array}{l}\text { These systems have the capabilities that enable humans to effectively complete surface } \\
\text { destination objectives, including human surface mobility, habitation and support } \\
\text { elements. }\end{array}$ \\
\hline & $\begin{array}{l}\text { Advanced In-Space } \\
\text { Propulsion }\end{array}$ & $\begin{array}{l}\text { In-space stage using nontraditional propulsion technologies, such as high power } \\
\text { electric and nuclear propulsion, to enable deep-space crew exploration of an asteroid } \\
\text { and Mars. }\end{array}$ \\
\hline
\end{tabular}

\section{Changes From GER Version 1}

The ISECG space agencies continue to review and refine their exploration plans, which are now reflected in GER version 2. The initial roadmap identified two potential pathways toward the driving goal of human exploration of Mars: "Asteroid Next" and "Moon Next." Each pathway was expanded through conceptual mission scenarios, which served as references to inform preparatory activities. Building on this work, the 2013 roadmap includes a single reference mission scenario that emphasizes the value of multiple destinations in a stepwise evolution of critical capabilities necessary for executing increasingly complex missions, leading to the human exploration of Mars. The roadmap demonstrates how initial capabilities can enable a variety of missions in the lunar vicinity, responding to individual and common goals and objectives, while contributing to building the partnerships required for sustainable human space exploration.

Human missions are initially focused on exploration of the lunar vicinity with opportunities to visit a small redirected asteroid or deploy an evolvable Deep Space Habitat. Other options would include Mars-preparatory exploration of the lunar surface. As reflected in Figure 1, these three options, or themes, are described in more detail 
in section IV. The robotic missions have also been updated to reflect agencies current and planned missions. Human-assisted sample returns are currently being assessed and human-scale EDL test mission opportunities are identified since advancements will be required for humans to explore the surface of Mars.

\section{Mission Themes}

The new version of the GER better reflects the multiple-pronged capability-driven approach to exploration advanced by NASA and other international space agencies, and described by one of the key GER driving principles. As capabilities evolve, a myriad of opportunities exist for agencies to explore together as partners. Each nation will have the opportunity to provide unique capabilities that serve to meet common beyond low Earth orbit (LEO) exploration goals, naturally building on the partnerships that enabled ISS. The use of mission themes rather than specific flights allows the capability to develop organically as the mission set evolves within the international community. While the mission themes certainly provide a means to confirm shared goals, they also can be aligned to target key technologies by providing test-beds for critical functions needed for future Mars missions and beyond.

To that end, three areas of commonality have been identified that can all contribute to building human exploration in a stepwise approach on the way to Mars and beyond. The three themes are: Exploration of a NearEarth Asteroid, Extended Duration Crew Missions in the lunar vicinity, and Humans Lunar Surface Missions (Figure 2). A stepwise approach must balance multiple needs and should strive to meet at least three conditions, which are combined aspects of the six principles already outlined: perform within the expected current or near-term capabilities, reduce mission risk by breaking up the advancing steps systematically, and feed forward directly to future exploration destinations, especially Mars. The final condition is articulated in more detail in Section V.

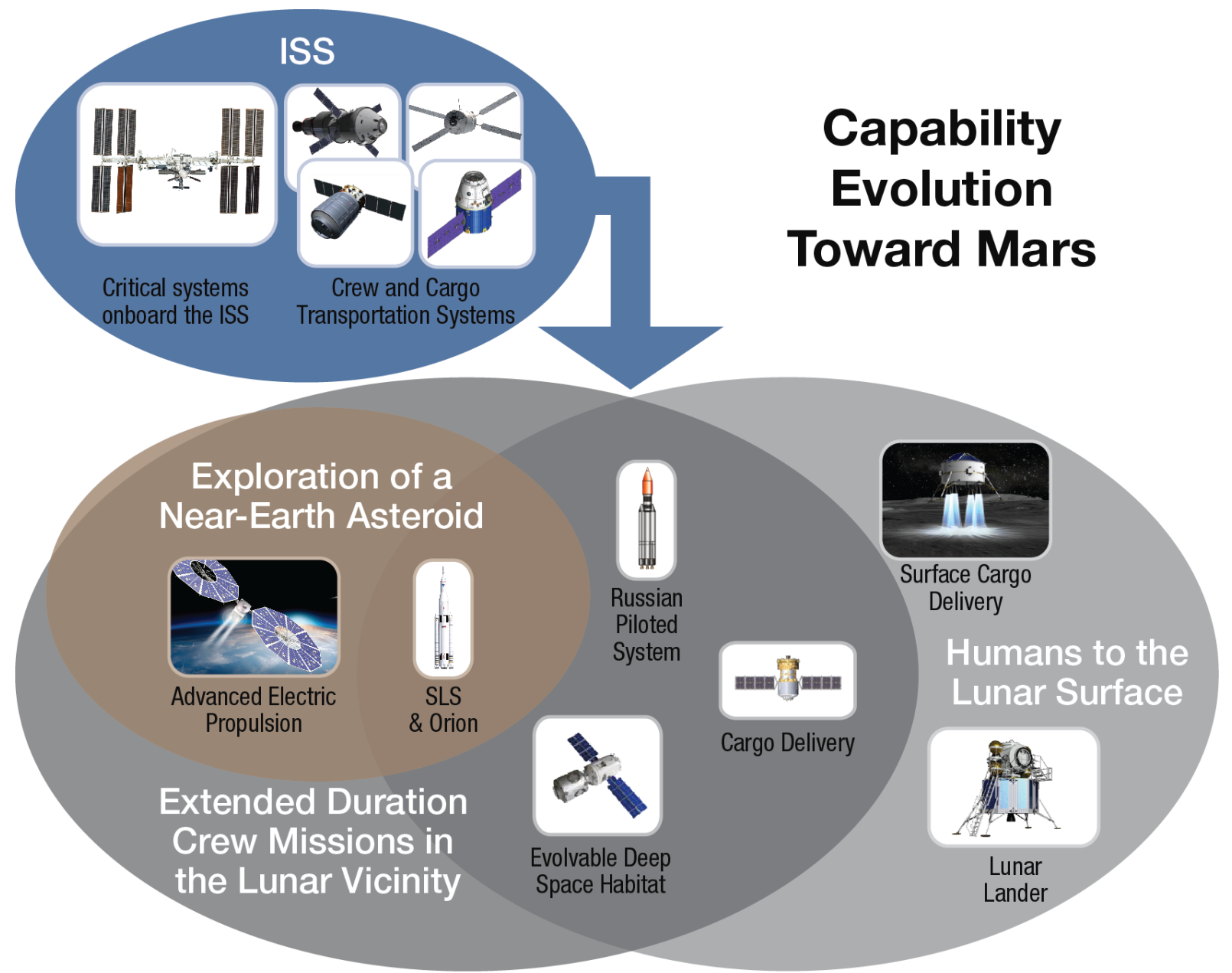

Figure 2. Capability Evolution Toward Mars 


\section{A. Exploration of a Near-Earth Asteroid}

The first theme, Exploration of a Near-Earth Asteroid, goes far in addressing two of the three conditions previously described. The NEA exploration mission does not require sending the crew to the asteroid but simply to the lunar vicinity, most likely in a Distant Retrograde Orbit (DRO) where a precursor robotic mission has already captured and pre-emplaced a small NEA of to-be-defined origin. The current Orion capsule and SLS Block 1 launch vehicle developments will be the first available elements and are the only required human transportation system components required for this mission theme. In addition this first beyond LEO step is relatively small with no significant technology developments and provides favorable opportunities for crewed mission aborts. The final condition is less ideally addressed, but some feed-forward activities are possible with the advent of unique extravehicular activities (EVA) tasks to dock to and examine the captured NEA. In addition, the robotic mission to retrieve the asteroid tests key technologies such as a large Solar Electric Propulsion (SEP) system that minimizes both mission and crew risk by testing a larger scale SEP before it is used in a human space mission.

\section{B. Extended Duration Crew Missions}

The second theme, Extended Duration Crew Missions, naturally ties into the NEA mission theme, but is not bound to it. By demonstrating advanced SEP on the asteroid robotic mission, a system of this size can be used on the DSH for repositioning and orbit maintenance. One of the biggest challenges for deep space exploration is long-term habitability in a harsh environment far from Earth. As humans venture further from the Earth, the very definition of space exploration, the spacecraft will need to shed its dependency on the resupply of critical consumables and services from Earth and instead rely on higher levels of reliability, autonomy, and efficiency. While the ISS provides many opportunities to test operations and technologies, the ISS is on Earth's doorstep and thus relies on regular deliveries from the surface for resupply and maintenance. The true test of advanced deep space system functions will be when rescue operations are much more limited. The best next step to prepare for these far flung long duration missions is within a stable environment far enough away from Earth to strain current operations, but not so distant to eliminate reasonable contingency scenarios. Placing an evolvable Deep Space Habitat (DSH) in the lunar vicinity is a good fit for this intermediate step. As a result, the extended duration theme also addresses all three conditions previously described. For initial capability, it might only require a single, possibly modular, element and the mission risk is lower compared to those in deep space. The rocket performance requirements can be constrained within planned capabilities for Orion and SLS and contingency aborts are present throughout. An initial capability of 90 days for baseline crew usage is deemed a reasonable lower limit that can be expanded as resources and technology tests dictate. Finally, there are a number of Mars feed-forward opportunities as addressed in Section V.

\section{Human Lunar Surface Missions}

The final theme, Human Lunar Surface Missions, again builds naturally on the previous theme, but like Extended Duration Crew Missions, can also be executed independently. Ultimately, the lunar lander concept of operations depends on whether missions to the lunar vicinity from the first two themes occur and if the deployed assets are reusable. If missions have already been conducted to the lunar vicinity, assets may have been left in a DRO or other stable orbit that could provide a staging point for missions to the surface. These assets could provide habitation, power, or even serve as a propellant depot for missions to the lunar surface, thereby reducing the overall delivery requirements to the lunar vicinity. Having a lower energy aggregation point than low lunar orbit (LLO) at EarthMoon Lagrange Point 2 (E-M L2) or at a DRO also enables access to smaller commercial or partner launch vehicles which could reduce the total number of SLS launches for a given lunar surface mission as well as increase the amount and frequency of cargo delivery to the surface. In addition, there is an opportunity to build reuse into the system. Additional analysis needs to be done to assess the likelihood of overall savings given the increased requirement and functionality burden these in-space elements would shoulder. The only new capability element required is a lunar lander, which can be modularized and/or made partially reusable. The optimal configuration ultimately would depend on the mission requirements, as determined by ongoing studies by interested agencies. The lunar surface provides significant Mars surface analog opportunities and if Mars focus is used to inform the lunar mission planning, could be a significant Mars risk reducer.

\section{Human Mars Mission Risk Reduction}

The three mission themes meet an evolving set of international capabilities with minimal near-term developments to ensure focus is spent on developing transportation elements. In the meantime, the near-term mission themes provide significant value to advancing human exploration towards Mars. Table 2 illustrates the applicability of the environments available for human testing for a complete Mars surface mission prior to 
Table 2. Mars Risk Reduction

\begin{tabular}{|c|c|c|c|c|c|c|}
\hline $\begin{array}{l}\text { - Full utilization in relevant environment } \\
\text { - Sufficient risk reduction in relevant environment } \\
\odot \text { Initial feasibility validation/partial validation }\end{array}$ & Earth & $\begin{array}{c}\text { ISS/Low-Earth } \\
\text { Orbit }\end{array}$ & $\begin{array}{c}\text { Lunar Vicinity } \\
\text { (Earth-Moon Lagrange } \\
\text { Point (EML), Moon Orbit) }\end{array}$ & $\begin{array}{l}\text { Moon } \\
\text { Surface }\end{array}$ & $\begin{array}{l}\text { Mars } \\
\text { Vicinity }\end{array}$ & $\begin{array}{c}\text { Mars Surface } \\
\text { (Robotic Mission) }\end{array}$ \\
\hline Beyond Low-Earth Orbit Crew Transportation & & & • & $\bullet$ & • & \\
\hline Heavy Lift Launch & & & $\odot$ & • & • & \\
\hline Reduced Supply Chain & & $\odot$ & • & • & • & \\
\hline Autonomous Crew Operations & $\odot$ & $\odot$ & $\bullet$ & $\bullet$ & $\bullet$ & \\
\hline Deep Space Staging Operations & & & $\bullet$ & & $\bullet$ & \\
\hline Mars Ascent & $\odot$ & & & $\odot$ & & $\odot$ \\
\hline Space Radiation Protection/Shielding & & $\odot$ & $\bullet$ & $\bullet$ & $\bullet$ & \\
\hline Life Support \& Habitation Systems & & $\bullet$ & $\bullet$ & $\bullet$ & $\bullet$ & \\
\hline Entry, Descent, \& Landing Systems & $\odot$ & & & $\odot$ & & $\bullet$ \\
\hline Surface Power and Energy Management & $\odot$ & & & $\bullet$ & & $\bullet$ \\
\hline Surface Mobility & $\odot$ & & & $\bullet$ & & $\bullet$ \\
\hline Human Robotic Integration & $\odot$ & $\bullet$ & $\bullet$ & $\bullet$ & $\bullet$ & $\bullet$ \\
\hline Mars In-Situ Resource Utilization & $\odot$ & & & $\odot$ & & $\bullet$ \\
\hline Long Duration Human Health & $\odot$ & $\bullet$ & $\bullet$ & $\bullet$ & $\bullet$ & \\
\hline Deep Space Operation Techniques & $\odot$ & $\odot$ & • & & • & \\
\hline
\end{tabular}

execution. The destinations are listed in the header from left to right in order of energy distance from Earth with key technologies and capabilities listed in the first column. The table is designed to provide information on how a risk reduction campaign could trace through intermediate destinations. To that end, while not every destination will necessarily be used to advance the maturity of a given technology on the left, where a dot exists the ability to at some level reduce the overall risk of a Mars mission is possible.

\section{A. Establishing Risk Reduction Categories}

Three categories of opportunity for risk reduction are given based on the applicability of the articulated destinations. They are:

1. Full utilization in a relevant environment: meaning an equivalent level of maturity can be conducted in this environment to completely match a Mars surface mission for a particular risk.

2. Sufficient risk reduction in a relevant environment: meaning most of a given risk could be reduced considerably but the analog environment has sufficient differences to prevent full validation.

3. Initial feasibility validation/partial validation: meaning some testing could be done but the risk would likely still be high due to the number of differences between the testing environment and the actual environment.

A good example of the capability to accomplish the largest level of risk reduction (Full Utilization) and at multiple deep space destinations is the Human Robotic Integration risk area. While Earth and ISS provide some level of capability to practice the anticipated human/robotic interaction needed for Mars missions, it is not sufficient to fully reduce the risk. However, a full-up Mars surface mission may not be necessary as the lunar environment permits operational activities to a degree largely commensurate with that which is currently required for Mars missions and the expected usage of in-situ low latency communication to facilitate human robotic interactions. For example, it is not a stretch to assume that tele-robotics from lunar orbit to the lunar surface has a substantially amount of similarity to tele-robotics of Mars assets from Mars orbit. The middle level of risk reduction (Sufficient Risk Reduction) is emulated at multiple destinations within the Life Support \& Habitation Systems development area. To demonstrate this system completely requires a space environment almost exactly equal to Mars. However, one of the biggest factors is time in a deep space environment, which makes the other locations, ISS, Lunar Vicinity and Moon Surface sufficient as locations to demonstrate a substantial amount of risk reduction. Even so, for some technologies, only Mars itself may be sufficient to reduce risk substantially and only the first level (Initial Feasibility) is available from a risk reduction viewpoint. The best example of the relative insufficiency of analogs is Entry, Descent \& Landing. Mars has an atmosphere unique unto itself. While the atmosphere can be simulated on computers, and concepts tested by matching some of the conditions in Earth atmosphere, large risks will exist until a full-scale entry vehicle descends into Mars. The entry event is dynamic with a number of critical maneuver 
sequences, pyros, propulsive firings, etc., all dependent on a correct understanding of the atmospheric composition and thickness, gravitational potential of Mars, and aerodynamic interaction of the entry vehicle.

\section{B. Destination Stepping Stones}

Examining the Mars risk reduction table from a destination perspective helps illustrate how a stepwise approach to deep space exploration is devised.

1. Earth

To start, the Earth column provides the basis for which initial technology development and fundamental research could be used to establish the building blocks for an exploration program. Breaking it down specifically for Mars needs, system analogs and testing exists to reduce risk in the following areas: Autonomous Crew Operations, Surface Power and Energy Management, Surface Mobility, Human Robotic Integration and Long Duration Human Health. Examples of useful analogs include recently operational activities such as NASA Extreme Environment Mission Operations (NEEMO) ${ }^{\text {iii }}$, NASA's Desert Research and Technology Studies (RATS) ${ }^{\text {iv }}$, and Russia and ESA's Mars $500^{v}$. In addition, basic research is instrumental in building the initial models to assess feasibility for Mars Ascent (systems testing for proposed propulsion systems such as liquid oxygen and Methane), Entry, Descent \& Landing Systems (detailed analysis and design of aeroshells in computer simulated Mars environment and subscale test flights), and Mars In-Situ Resource Utilization (scale model tests using simulated Mars regolith).

\section{ISS/Low-Earth Orbit}

For ISS, a mostly different set of technologies can be advanced and demonstrated. It is unlikely that fundamental research into key Mars technologies such as power or mobility or ISRU would make efficient use of the unique characteristics of the ISS laboratory. On the other hand, ISS is a perfect place to begin to examine how to reduce risks associated with Life Support \& Habitation Systems, Space Radiation Protection/Shielding and Long Duration Human Health. In fact, there are upcoming plans for up to one-year missions to ISS to evaluate these issues. Likewise, while Earth is not far away, there is an opportunity to begin to examine the effect of Reduced Supply Chain and Autonomous Crew Operations by reducing reliance on cargo resupply missions by evolving the systems to be autonomous and regenerative.

\section{Lunar Vicinity (Earth-Moon Lagrange Points, Moon Orbit)}

Progressing from LEO to near the Moon expands the opportunity to demonstrate Mars technologies substantially. All of the areas that could be initially tested on ISS would provide more substantial feed-forward to Mars risk reduction results in the lunar vicinity. The lunar vicinity offers a much higher energy state to begin testing the beyond LEO transportation system. For NASA this means the first true utilization of the SLS/Orion system. Perhaps the most compelling usage of the lunar vicinity is as a deep space staging point. It is clear from Mars mission studies that multiple launch vehicles will be needed and that aggregation of assets, both cargo and crew is needed. The lunar vicinity presents a unique environment to develop these capabilities and address the operations concerns and uncertainties associated with aggregations such as autonomous rendezvous and docking (AR\&D).

\section{Moon Surface}

The best analog away from a Mars mission itself is a human lunar surface landing. The next column in the Mars risk reduction table demonstrates this fact. Aside from Deep Space Staging, which is the largest advantage of the lunar vicinity destination, all rows have some level of opportunity for risk reduction to Mars. It is important to note, however, just like the other destinations, Mars mission risk reduction is only realizable if aspects of the mission are designed to test the technologies and operations that will be conducted at Mars. Certainly missions at all destinations can be designed where substantial Mars mission risk reduction is not realized. A trade between the impact of adding mission critical functionality designed for risk reduction of future missions compared to a minimal set of requirements designed to successfully complete an intermediate missions on time and on-budget will be required.

5. Mars Vicinity and Mars Surface (Robotic Mission)

The final two categories will provide the remaining amount of risk reduction as desired for human missions to the Mars surface. Mars vicinity includes Mars orbit, as well as Mars moons, Phobos and Deimos. For these missions all of the non-surface related challenges could be fully investigated, while the surface requirements could be assessed to at least some level with robotic missions. The five challenges that will likely have some risk (i.e. no opportunity for full utilization within the intermediate destination context) prior to a human Mars surface mission are: Mars Ascent, EDL, Surface Mobility, Mars ISRU and Long Duration Human Health. Of these the largest risks with the least amount of pedigree with the least amount of flexibility will be Mars Ascent and EDL. Thus a Mars mission might very well consider performing these events at the human scale with cargo instead of crew to reach acceptable risk levels prior to a full-up crew mission to and from the Martian surface. 


\section{Summary}

The updates in the latest version of the GER reflect important steps in achieving a global, strategic, coordinated, and comprehensive approach to space exploration. Specifically, updates to the approach depicted in the ISECG mission scenario reflect efforts agencies have made to respond to stakeholder feedback from the global space community since the release of version 1 of the GER in September 2011. While the mission scenario does not create specific commitments on behalf of the participants, it does provide a common framework for agencies to discuss common goals and objectives and advance individual efforts in exploration planning, investment, and development. With ever-changing budgetary and political environments at the national level, agencies have acknowledged the importance of international participation to enable a sustainable future for space exploration. A key outcome has been the realization that investments made in exploration capabilities must be applied in a manner which is dictated by a stepwise approach to ever increasing capabilities to venture increasingly further away from Earth, for longer periods of time, as we collectively endeavor to eventually expand human presence to Mars.

\section{Acknowledgments}

The authors would like to acknowledge the following principle participants in the ISECG International Architecture Working Group (IAWG), including

- Yves Gonthier and Eric Martin, representing the Canadian Space Agency (CSA)

- Bernhard Hufenbach and Alessandro Bergamasco, representing the European Space Agency (ESA)

- Naoki Satoh, Hiroshi Ueno, and Toshiki Morito, representing the Japanese Aerospace Exploration Agency (JAXA)

- Kathy Laurini, Brenda Ward, John Connolly, Kevin Watts, Patrick Troutman, Daniel Mazanek, Kevin Larman, and Michelle Rucker representing National Aeronautics and Space Agency (NASA)

- Dmitry Zarubin, George Karabadzhak, and Oleg Saprykin, representing Roscosmos.

\section{References}

\footnotetext{
${ }^{\mathrm{i}}$ International Space Exploration Coordination Group, “The Global Exploration Roadmap," September 2011.

${ }^{\text {ii }}$ Cublert, C., Mongrard, O., Satoh, N., Goodliff, K., Seaman, C., Troutman, P., Martin, E., "ISECG Mission Scenarios and Their Role in Informing Next Steps for Human Exploration Beyond Low Earth Orbit," IAC-11-D3.1.2, $62^{\text {nd }}$ International Astronautical Congress, Cape Town, South Africa, 2011.

iii http://www.nasa.gov/mission_pages/NEEMO/

${ }^{\text {iv }} \mathrm{http} / / / \mathrm{www} \cdot$ nasa.gov/exploration/analogs/desertrats/

${ }^{v}$ http://www.esa.int/Our_Activities/Human_Spaceflight/Mars500
} 